\title{
INFORMACIÓN AUMENTADA CON MÓVIL EN MUSEOS MEDIANTE LOCALIZACIÓN CON BLE
}

\author{
Nuria Ortega-Sánchez, Antonio R. Jiménez, Fernando Seco \\ Centro de Automática y Robótica (CAR), Consejo Superior de Investigaciones Científicas (CSIC)-UPM \\ Ctra. Campo Real km 0.2, 28500 La Poveda, Arganda del Rey, Madrid, España. \\ e-mail: n.ortega@alumnos.upm.es, \{antonio.jimenez, fernando.seco\}@csic.es \\ web: http://www.car.upm-csic.es/lopsi
}

\section{Resumen}

Presentamos el diseño y desarrollo de una solución para el guiado contextualizado durante visitas a museos. Para ello se han distribuido por el entorno balizas de tipo Bluetooth Low Energy (BLE), de las cuales el smartphone lee las medidas de potencia recibida ( $R S S)$, junto con información de los sensores de acelerómetro y magnetómetro. Con la información sensorial y la del contexto, previamente almacenada en la nube, la app estima la ubicación de la persona en el recinto del museo con sus diferentes salas, y es capaz de calcular la distancia y la orientación de la persona respecto a los cuadros de interés. La precisión en la capacidad para medir estos parámetros es analizada utilizando diferentes métodos de trilateración usando filtros de Kalman extendidos (EKF) con integración débil y acoplada. Los resultados de localización obtenidos nos permiten tener suficiente precisión como para poder guiar al usuario en búsqueda de un cuadro o ruta de cuadros recomendada, o también para dar la información del cuadro más cercano en la dirección de visualización de usuario. El potencial uso e impacto de este enfoque se puede extender a otras aplicaciones en sectores industriales o robóticos que requieran asistencia o guiado en la búsqueda de objetos.

Palabras clave: Localización, Fuerza de señal recibida, Estimación Bayesiana, balizas BLE

\section{Introducción}

Las técnicas de localización en entornos interiores proporcionan un gran potencial en múltiples aplicaciones. Existen aplicaciones en el mundo industrial, logístico, de espacios inteligentes, marketing, guiado de personas, seguridad y emergencias, así como aplicaciones más cotidianas para personas usando tecnología estándar. La existencia de teléfonos móviles con capacidades de procesamiento y sensoriales sin precedentes, hace posible la creación de soluciones personales de valor añadido. Entre estas aplicaciones novedosas están las ligadas a la información aumentada, como en guias basadas en teléfono móvil para museos.
En el dominio de soluciones basadas en móviles y apps para museos podemos citar varios trabajos de investigación que usan marcas en el entorno, etiquetas RFID o códigos QR, para que el usuario se aproxime a unos pocos centímetros del marcador en la pieza de museo para obtener la información aumentada deseada $[11,10]$. Para evitar movimientos forzados del usuario con su móvil, otros trabajos han creado identificadores de presencia locales pero de mayor alcance [3], destacando los trabajos que usan ultrasonidos $[2,1]$. En ellos unos emisores locales de ultrasonidos (frecuencias $>18$ $\mathrm{kHz}$ ) generan códigos únicos de identificación permitiendo al téléfono móvil detectar que está en la zona de influencia de un objeto del museo, y gracias a ello proporcionar la información aumentada. Más deseable aún que estas soluciones son aquellas en las que el usuario podría localizarse de forma continua dentro del museo, y en base a la ubicación de las obras de arte, poder proporcionar no solo la información de la pieza sino otras funcionalidades como la navegación o guiado a una obra de interés. En este sentido ya se han empezado a desarrollar soluciones comerciales que tratan de explotar este concepto de guiado y localización continua (http://www.gipstech.com y www.stendhal.com) en museos.

Diferentes enfoques se han propuesto para el guiado o localización continua de personas con balizas y teléfonos móviles $[9,6]$. Cabe destacar aquellos usando la tecnología Bluetooth Low Energy (BLE), la cual es muy popular hoy en día desde la definición del protocolo estándar iBeacon $[8,5]$. Con BLE las etiquetas o balizas emiten a intervalos regulares un código de identificación, así como otros parámetros de interés (movimiento, temperatura, presión, etc.). El rango o distancia se puede deducir o estimar con la potencia de la señal recibida (RSS) mediante un modelo de pérdidas de potencia con la distancia de propagación de la señal de radio. Sin embargo, a diferencia de técnicas basadas en UWB donde la precisión es submétrica, en nuestro caso al trabajar con potencia de la señal (caso de BLE, pero también de Wifi), la precisión obtenida en medidas de rango suele estar entre 3 y 5 metros, especialmente cuando la distancia es superior a unos pocos metros. Sin embargo, a cortas 
distancias, lo cual se consigue aumentando la densidad de emisores, el método de posicionamiento basado en RSS sí que es capaz de mostrar precisión válida para muchas aplicaciones, y que suelen estar en el rango de 1-3 metros [4].

En este trabajo presentamos el desarrollo de una App en Android, que utilizando información BLE para el posicionamiento, permite el guiado y orientación durante visitas a museos, y la provisión de información ampliada acorde con el contexto (en nuestro caso la de un museo y la ubicación y detalles inportantes de un cuadro). Para ello se han distribuido por el entorno balizas de tipo Bluetooth Low Energy (BLE), de las cuales el smartphone lee las medidas de potencia recibida (RSS), junto con información de los sensores de acelerómetro y magnetómetro. La información del museo, previamente editada mediante un modo de desarrollador en la app, se almacena en la nube en un servidor. Con la información sensorial y la del contexto, la app estima la ubicación de la persona en el recinto del museo con sus diferentes salas, y es capaz de calcular la distancia y la orientación de la persona respecto a los objetos de interés (cuadros en el caso del museo). El potencial uso e impacto de este enfoque se puede extender a otras aplicaciones en sectores industriales o robóticos que requieran asistencia o guiado en la búsqueda de objetos.

El diseño de la App y su forma de uso se presenta en la sección 2. La precisión que hemos obtenido a la hora de estimar la posición del usuario con el móvil se analiza utilizando diferentes métodos de trilateración usando filtros de Kalman extendidos (EKF) con integración débil y acoplada. Los resultados de posicionamiento, medida de rangos a los cuadros y orientación usuario/cuadro se muestran en la sección 3. Finalmente damos unas conclusiones.

\section{Diseño y funciones de la App}

\subsection{Perfiles de uso de la app}

Esta aplicación tiene dos modos de funcionamiento para los distintos perfiles de usuario: perfil de visitante del museo y perfil de desarrollador.

\subsubsection{Perfil de desarrollador}

Este perfil es para los gestores de cada museo registrado, quienes tendrán usuario y contraseña propio. Al modo desarrollador se accede desde la pantalla principal, a través de un login en el cajón de navegación.

Para que el modo visitante esté operativo en un determinado museo, primero cada museo ha de ser configurado por el "desarrollador", el cual introducirá los datos necesarios: salas, dimensiones, orientación, ubicación de cuadros y balizas. Una vez introducidos los datos básicos, se procede a determinar los parámetros óptimos para la localización en el interior de edificios. Estos parámetros serán usados tanto en la ecuación de Path Loss como en los filtros de Kalman.

Desde el perfil desarrollador, se generarán los ficheros que almacenan la información necesaria durante el proceso de pruebas de posicionamiento para exportarlos a Matlab y proceder a la obtención de los parámetros. En total, la aplicación en perfil desarrollador cuenta con 3 algoritmos de posicionamiento: trilateración, trilateración + filtro de Kalman (Kalman desacoplado) y filtro de Kalman integrado. El motivo de incorporar estos 3 tipos de posicionamiento es el de realizar un estudio comparativo entre ellos y justificar así el uso del mejor de ellos en las actividades de la app del perfil del visitante. Cuando el desarrollador termina de configurar un museo debe subir toda la información actualizada al servidor público de nuestro centro CAR desde la propia aplicación. Es importante destacar que estas acciones se deben repetir para cada museo que funcione con esta aplicación.

\subsubsection{Perfil de visitante del museo}

El perfil de visitante del museo está disponible desde el momento en que se abre la aplicación: en la pantalla principal aparecen todas las acciones con las que se puede interactuar. El contenido está organizado a través de una vistosa lista de botones (Fig.1) con un breve resumen del contenido al que se accede a través de ellos. El uso de la app comenzaría pulsando el botón "Bienvenido" en primer lugar, el cual borrará la base de datos local de la aplicación y se descargará otra actualizada del servidor refrescando la información del museo en el que nos encontremos.

Con el fin de no saturar el almacenamiento del dispositivo, los datos de los museos se almacenarán en un servidor, y se descargarán cuando sea estrictamente necesario solo aquellos datos acordes al contexto y no almacenando la información de otros museos que se hayan visto con anterioridad. Además, al descargar la información se le ofrece al cliente la versión más actualizada de las exposiciones.

El criterio que se ha seguido para determinar el contexto y decidir en tiempo real cuál es el museo que se está visitando, reside en la lectura de un parámetro de la señal de las balizas de BLE llamado "major" presente en el identificador de la señal. Por tanto hacemos uso del nuevo concepto de "Web Física". Las balizas BLE usadas 


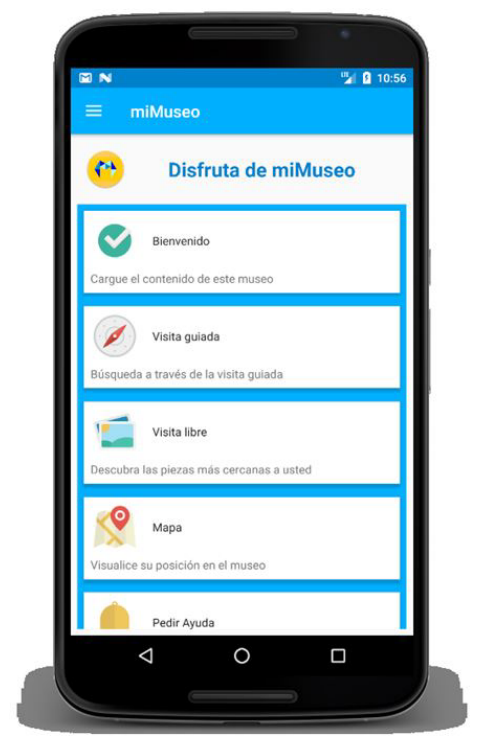

Figura 1: Pantalla principal de la App de Android en el modo visitante.

están configuradas para utilizar el protocolo iBeacon para la transmisión de paquetes Bluetooth y emiten a una frecuencia configurable una cantidad pequeña de datos entre los que se encuentra el identificador de la baliza. Este identificador está formado por 3 partes: "UUID", "major", y "minor" los cuales se pueden personalizar. Asignando a todas las balizas de un mismo museo un mismo valor de "major" podremos saber en qué museo nos encontramos. Cabe destacar que el contenido de un museo es accesible a través de la app solo cuando el usuario se encuentra en dicho museo, pues el objetivo de la app es el posicionamiento y guiado del usuario dentro del museo.

Siguiendo con las opciones disponibles desde el perfil de visitante del museo, una vez descargado el contenido del museo, podemos acceder al contenido del resto de los botones de la pantalla principal (Fig.1) ya que se acabarían de desbloquear. Ahora se tendría permiso para usar "Visita Guiada", "Visita Libre", "Mapa" y "Pedir Ayuda":

- Visita Guiada. Le muestra al usuario una lista con todos los cuadros del museo que han sido subidos al servidor. Una vez se elige uno, la aplicación a través de una pequeña "brújula de cuadros" indica la dirección en la que se encuentra dicho cuadro, la distancia a la que está del usuario y contenido web con la información ampliada del cuadro (Fig.2).

- Visita Libre. Es una elección más relajada en la que es el propio usuario el que decide cómo moverse por el museo, y cuando encuentre una obra que le interese, puede pulsar en el botón flotante de actualizar contenido web y

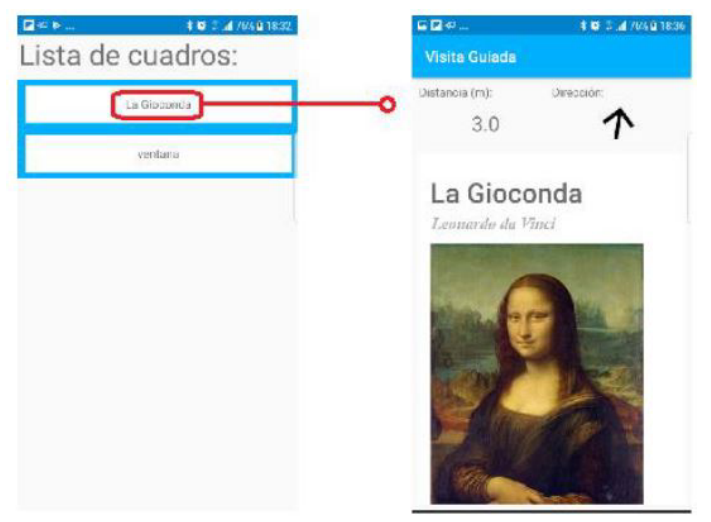

Figura 2: Pantalla de visita guiada (o de búsqueda de cuadros sugeridos) en la App de Android.

se le mostrará en la pantalla la información ampliada de la pieza. Solo da información de la obra más cercana si el usuario se encuentra en la misma sala que la obra.

- Mapa. Refleja la posición del usuario en un pequeño canvas de la sala en que se encuentra el usuario y el número de la sala (Fig.6). Su finalidad es ayudar al visitante a ubicarse en el museo.

- Pedir Ayuda. Sitúa al usuario en el museo y envía un mensaje al personal del museo para que acuda a dicha posición. La finalidad de esta opción es amplia, puede ser útil desde ayudar al visitante con alguna duda hasta alertar de una emergencia.

"Visita Guiada" es una de las ofertas de la aplicación para que el visitante encuentre fácilmente las obras que le interesen. Para llegar a esta actividad (Fig. 2), pulsamos sobre el botón de la pantalla principal "Visita Guiada" y ésta nos llevará a una pantalla con una lista de todos los cuadros con información ampliada de que dispone el museo. Pulsando sobre uno de los cuadros accederemos a la actividad "Visita Guiada".

Una ventaja de esta app es que podemos configurar múltiples museos y los visitantes visitarlos cuando deseen teniendo información permanentemente actualizada desde la misma aplicación instalada inicialmente.

A pesar de que esta aplicación ha sido planteada para museos, su uso podría extenderse a otros edificios públicos de grandes dimensiones como aeropuertos, hospitales, centros comerciales... ayudando a los ciudadanos a orientarse y proporcionarles información de utilidad. 


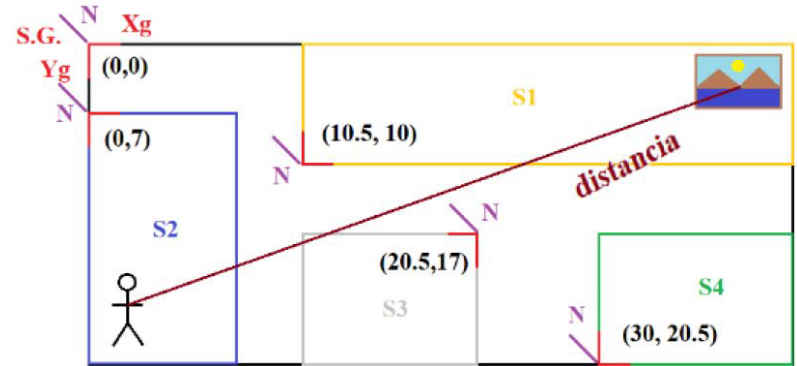

Figura 3: Definición del mapa del museo y sistemas de referencia.

\section{Resultados de posicinamiento $\mathrm{y}$ guiado}

En esta sección mostramos cómo se realiza el posicionamiento del usuario dentro del museo, y cómo a partir de esa estimación es posible estimar la distancia y orientación entre el usuario y la pieza de museo deseada.

\subsection{Sistema de referencia y mapeado}

En primer lugar se define el mapa del museo que consiste en un conjunto de salas rectangulares. Cada sala tiene unas dimensiones y una orientación espacial. En la Fig. 3 se ve un ejemplo ilustrativo con 4 salas (S1, S2, S3 y S4). Cada sala tiene un sistema de referencia local X-Y y su orientación respecto al norte magnetico terrestre $(\mathrm{N})$. Cada ubicación de los cuadros es relativa al sistema de referencia local de cada sala, y las balizas BLE que se coloquen en cada sala también están referenciados localmente. Además hay un sistema de referencia global (S.G.) que se usa para tener un marco común de referencia cuando la localización implica elementos en distintas salas (p.ej. un cuadro en una sala distinta a donde está el usuario).

\subsection{Conversión de RSS en rango}

Para localizar al usuario es común usar técnicas de trilateración una vez que se disponen de varios rangos a balizas. Debido a que las medidas BLE disponibles en el móvil consisten en la potencia recibida (RSS) de cada una de las balizas en proximidad, es necesario tratar de estimar este rango, para lo cual usamos un modelo de atenuación de la señal con la distancia (path-loss model). Este modelo transforma cada RSS en distancia, $d$, tal y como se hace en [7]:

$$
d=d_{0} \cdot 10^{\frac{\mathrm{RSS}_{0}-\mathrm{RSS}}{10 \cdot p}} .
$$

donde $\mathrm{RSS}_{0}$ es un valor medio de RSS obtenido a una distancia de referencia $d_{0}$, y $p$ es el exponente de pérdidas que para la mayoría de nuestros experimentos usando las balizas Estimote es de $\mathrm{RSS}_{0}=-60 \mathrm{~dB}, d_{0}=1 \mathrm{~m}$ y $p=1,6$. La desviación estándar de la distancia estimada en eq. 1 la designamos $\sigma_{d}$, la cual es necesitada para indicar la incertidumbre que tenemos sobre la medida cuando se integre en el filtro de Kalman. Esta incertidumbre depende de la distancia y se calcula así:

$$
\sigma_{d}=\sigma_{\mathrm{RSS}} \cdot \frac{\ln (10) \cdot d}{10 \cdot p}
$$

Este modelo sigma es proporcional al ruido de las medidas RSS $\sigma_{\mathrm{RSS}}$, y a la distancia, por tanto proporciona una baja desviación estándar a cortas distancias (baja incertidumbre) y un sigma mayor a distancias largas (mayor incertidumbre).

\subsection{Algoritmos de localización con rangos}

Una vez transformados los valores RSS en rangos (tal y como se describió en el apartado anterior), pasamos a realizar la trilateración con ellos para obtener la ubicación del usuario y su móvil. Para ello utilizamos un filtro de Kalman extendido (EKF) [7], que hará las labores de trilateración y suavizado predictivo, usando 4 estados que representan la posición y velocidad:

$$
\mathbf{X}=\left(x, y, v_{x}, v_{y}\right)
$$

donde $(x, y)$ es la posición del móvil en el sistema global, y $\left(v_{x}, v_{y}\right)$ es la velocidad. Los modelos de movimiento elegidos y los de medida son fundamentales para obtener una estimación con retardos tolerables y suficientemente libres de ruido (trayectorias suaves). Se describen a continuación estos modelos.

\subsubsection{Modelo de movimiento}

El modelo de movimiento utilizado es uno de velocidad constante con cambios de velocidad de la persona caminante modelados como ruido de aceleración $\left(g_{a}(a)\right)$ :

$$
\mathbf{X}=f\left(x_{c}, y_{c}, v_{x}, v_{y}\right)+g_{a}(a)
$$

El modelo en tiempo discreto, con un intervalo de muestreo $d t$, sería: 


$$
\mathbf{X}^{k+1}=\left(\begin{array}{c}
x_{c}^{k+1} \\
y_{c}^{k+1} \\
v_{x}^{k+1} \\
v_{y}^{k+1}
\end{array}\right)=\left(\begin{array}{l}
x_{c}^{k}+v_{x}^{k} d t+\frac{a^{k} d t^{2}}{2^{2}} \\
y_{c}^{k}+v_{y}^{k} d t+\frac{a^{k} d t^{2}}{2} \\
v_{x}^{k}+a^{k} d t \\
v_{y}^{k}+a^{k} d t
\end{array}\right)
$$

de donde se deduce que la matriz de transición de estados del filtro de Kalman es

$$
F=\left(\begin{array}{llll}
1 & 0 & d t & 0 \\
0 & 1 & 0 & d t \\
0 & 0 & 1 & 0 \\
0 & 0 & 0 & 1
\end{array}\right)
$$

Si definimos $G_{a}=\left[\frac{d t^{2}}{2}, \frac{d t^{2}}{2}, d t, d t\right]^{\prime}$ podemos representar la matriz de covarianza del proceso o modelo de movimiento a utilizar en el EKF de esta manera:

$$
Q=G_{a} q_{a} G_{a}^{\prime}=\left(\begin{array}{llll}
\frac{d t^{4}}{4} & 0 & \frac{d t^{3}}{2} & 0 \\
0 & \frac{d t^{4}}{4} & 0 & \frac{d t^{3}}{2} \\
\frac{d t^{3}}{2} & 0 & d t^{2} & 0 \\
0 & \frac{d t^{3}}{2} & 0 & d t^{2}
\end{array}\right) q_{a}
$$

donde $q_{a}$ es la covarianza del ruido en aceleración que depende de cómo se mueva la persona, y cómo difiere su modo de caminar de un movimiento con velocidad constante.

\subsubsection{Modelo de medida}

El modelo de medida representado por la matriz $H$ relaciona los estados con las medidas (rangos). Como en nuestro caso el estado es posición y las medidas son rangos existe una relación no lineal que se ha de linealizar, y que supone un proceso interno de trilateración. Nuestra matriz $H$ viene dada por la siguiente expresión:

$$
H=\left(\begin{array}{llll}
\frac{x^{1}-\hat{x}}{\hat{r}^{1}} & \frac{y^{1}-\hat{y}}{\hat{r}^{1}} & 0 & 0 \\
\frac{x^{2}-\hat{x}}{\hat{r}^{2}} & \frac{y^{2}-\hat{y}}{\hat{r}^{2}} & 0 & 0 \\
\cdots & \cdots & 0 & 0 \\
\frac{x^{i}-\hat{x}}{\hat{r}^{i}} & \frac{y^{i}-\hat{y}}{\hat{r}^{i}} & 0 & 0 \\
\cdots & \cdots & 0 & 0 \\
\frac{x^{N}-\hat{x}}{\hat{r}^{N}} & \frac{y^{N}-\hat{y}}{\hat{r}^{N}} & 0 & 0
\end{array}\right)
$$

donde $H$ es una matriz de dimensión variable con tantas filas como rangos $N$ medidos (o número de RSS procedentes de distintas balizas BLE) y que tiene tantas columnas como los estados estimados (4); $\hat{r}^{i}$ es el rango entre la baliza $i$ y la última posición estimada del móvil, $(\hat{x}, \hat{y})$, es decir: $\hat{r}^{i}=$ $\sqrt{\left(\hat{x}-x^{i}\right)^{2}+\left(\hat{y}-y^{i}\right)^{2}}$.

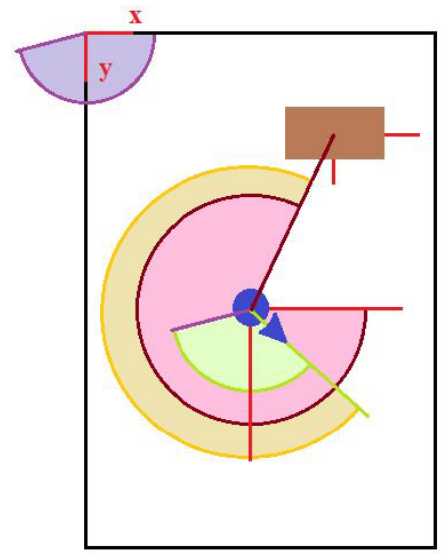

Figura 4: Definición de los ángulos de referencia.

\subsection{Cálculo de la orientación usuario-cuadro}

Una vez que conocemos con cierta precisión la ubicación del usuario mediante el filtro de Kalman presentado anteriormente, y sabiendo la ubicación de los cuadros, es posible saber la orientación entre cuadro y el usuario. Además gracias a la medición de la brújula electrónica del móvil, es posible dar una indicación en la pantalla del móvil de hacia dónde está el cuadro buscado respecto a la pantalla del móvil.

En la Fig. 4 se esquematiza un supuesto visitante mirando hacia la esquina inferior derecha, representado por un punto azul con flecha; las sombras sectoriales de colores representan los diferentes ángulos que participan en el proceso de cálculo de la orientación. El rectángulo marrón representa el cuadro que se está buscando. El semi arco verdoso representa la orientación medida por la brújula electrónioca que indica la desviación respecto al norte. El ángulo rosa es el ángulo geométrico que surge al oberservar las posiciones de la persona y el cuadro respecto al eje X, y que también se puede calcular respecto al norte. El águlo externo color crema, representa la orientación que estamos buscando y que indica respecto a la orientación específica del móvil hacia dónde debe mirar o dirigirse la persona para encontrar el cuadro.

\subsection{Errores de posicionamiento}

En este apartado queremos mostrar los resultados experimentales de localización realizados en un entorno representativo de prueba. En el experimento se ha creado una malla de puntos en posiciones conocidas donde una persona se ha posicionado durante cierto tiempo (60 segundos) y realizado una rotación de $360^{\circ}$. El motivo del giro es para generar diversidad en la atenuación de las medidas debido a la atenuación del cuerpo de la persona, 


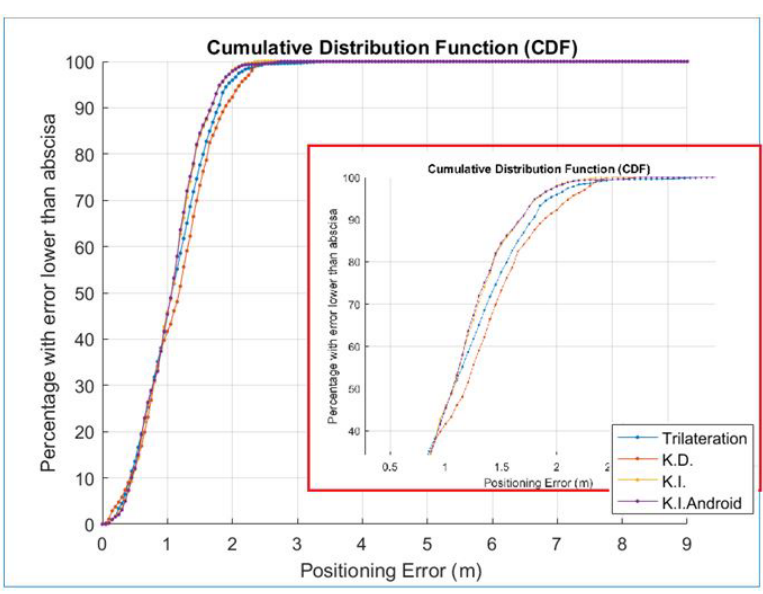

Figura 5: Error acumulativo de posición para tres algoritmos d eposicionamiento: Trilateración, K.D. y K.I.

y así hacerlo más realista.

Respecto al método de localización a evaluar, cabe mencionar que no solo se ha hecho con el explicado arriba sino que hemos ensayado otros enfoques alternativos más directos: Trilateración y Kalman directo (K.D). En el primer caso, no se usa la historia pasada para localizar, solo las medidas en un instante dado (es decir sin modelo de movimiento), y se require un mínimo de tres rango para poder dar una solución de posición. En el segundo caso (K.D.), se usa la trilateración previa sin historia para obtener la ubicación, y posteriormente se usa un filtro de Kalman que mediante un modelo de medida lineal suaviza las posiciones de entrada, generando una salida de posicionamiento suavizada. El filtro presentado en este paper es más potente ya que hace una trilateración integrada, que entre otras ventajas permite realizar estimaciones incluso con pocos rangos disponibles (incluso con una sola medida, cuando una trilateración estándar necesita al menos 3 rangos para obtener solución).

Los resultados tal y como se muestran en la figura 5, mediante una función de distribución de error acumulativa (CDF), indican que el método con mejores resultados es el integrado descrito en este artículo. Los errores de posicionamiento son de 1.5 metros en el $90 \%$ de los casos. Estos resultados hacen que en un museo típico, sea posible distinguir con cierta precisión la dirección del cuadro de interés, siempre y cuando la distancia a este objeto sea superior a 2-3 metros, ya que en caso contrario el error de posicionamiento enmascararía la medida correcta de la orientación.

Aunque la App no está fundamentalmente diseñada con la idea de mostrar la ubicación al usuario, sin embargo en el modo de desarrollador es po-

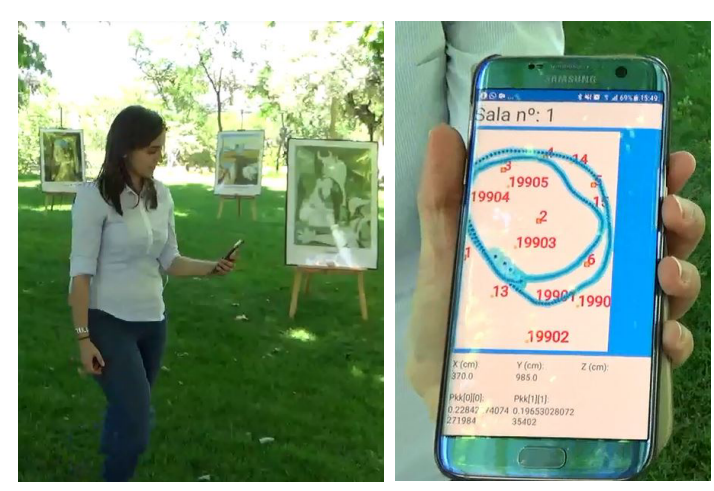

Figura 6: Traza de dos vueltas a una trayectoria circular en un museo al aire libre. La traza se muestra sobre el mapa del museo (con marcas de balizas BLE y ID de cuadros).

sible ver la ubicación del usuario en tiempo real, la incertidumbre (radio de una esfera), y la traza de la trayectoria recorrida. Para dar una idea de la calidad y suavidad de las estimaciones mostramos, en la Fig. 6, la traza para una trayectoria realizada en unos tests con un museo en el exterior.

\subsection{Errores angulares}

En este apartado mostraremos los resultados experimentales de medida de orientación para el mismo caso de prueba anterior. Si analizamos directamente el error en las estimaciones de orientación, en comparación con la orientación verdadera (Fig. 7 arriba) y también mediante otra CDF (en la parte baja de la misma figura 7), podemos concluir que en el $90 \%$ de los casos el error angular es menor de 50 grados. Esta cifra que no es del todo baja, sin embargo es suficientemente precisa como para poder saber si un cuadro está enfrente, detrás, a la izquierda o derecha de la persona. Según se vaya moviendo la persona y reorientando, esta estimación se va actualizando ayudando a dirigirse al sitio correcto. Como se ha dicho antes, existe la limitación de que si se aproxima uno demasiado al cuadro la orientación ya no será precisa. En este extremo caso de proximidad absoluta, entendemos que no es necesario tener precisión pues el cuadro está evidentemente a la vista del usuario y ya no necesita ayuda de orientación de la App para encontrarlo.

\subsection{Información aumentada en el móvil}

Aquí queremos ilustrar con las imágenes capturadas de algunos de los tests realizados (Fig. 8) cómo la información aumentada se visualiza sobre el móvil para proporcionar datos de interés al usuario.

La información por proximidad funciona correcta- 

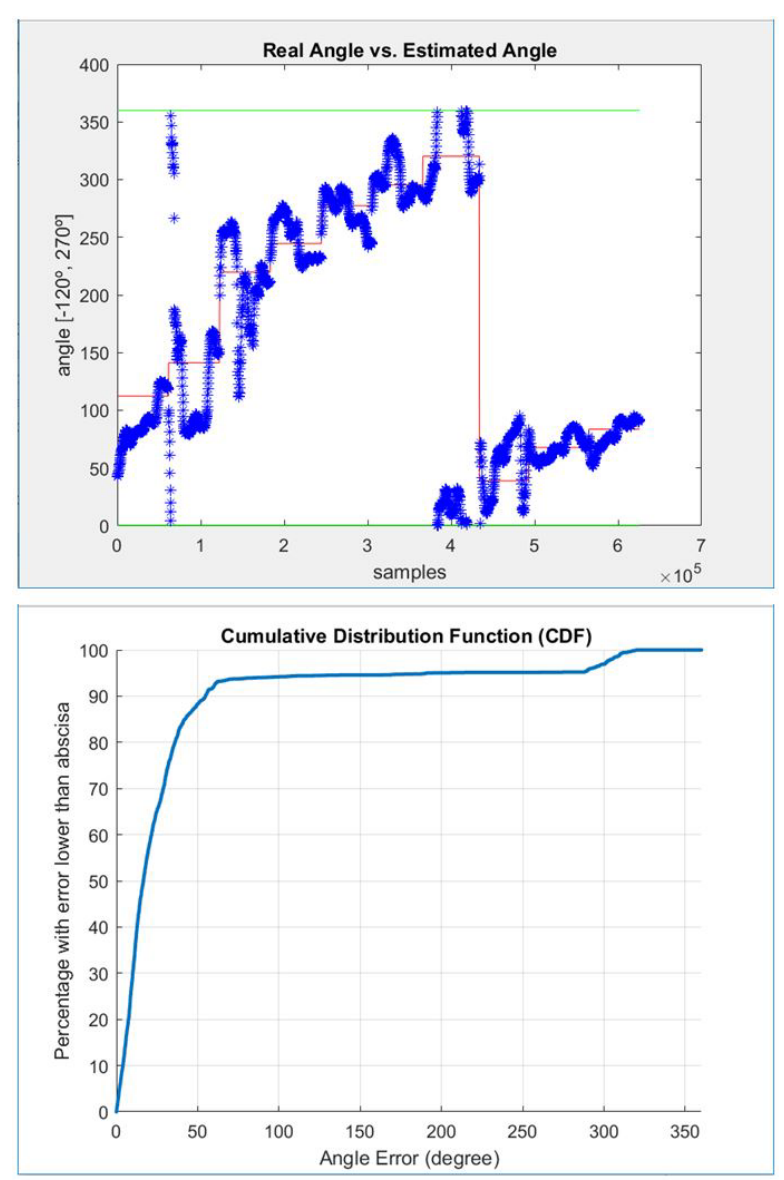

Figura 7: Ángulos estimados versus valores angulares verdaderos (arriba). Error acumulativo de orientación con el mejor algoritmo de posicionamiento (K.I.).

mente la gran mayoría de los casos, con el único requisito que la densidad de los cuadros no sea muy alta. Si los cuadros están separados más de 2 metros no suele haber problemas de identificación.

\section{Conclusiones}

Hemos presentado una aplicación para móvil que es capaz de definir información contextual basada en ubicación dentro de diferentes salas de un museo. La correcta generación de la información añadida (guiado distancia/orientación o la descripción ampliada) depende en gran medida de la calidad de la localización de la persona en el museo. Los resultados de localización obtenidos nos permiten tener suficiente precisión como para poder guiar al usuario en búsqueda de un cuadro o ruta de cuadros recomendada, o por el contrario dar la información del cuadro más cercano en la dirección de visualización de usuario. Se demuestra la utilidad de la tecnología de balizas BLE para este tipo de caso de uso u otras áreas de aplicación.
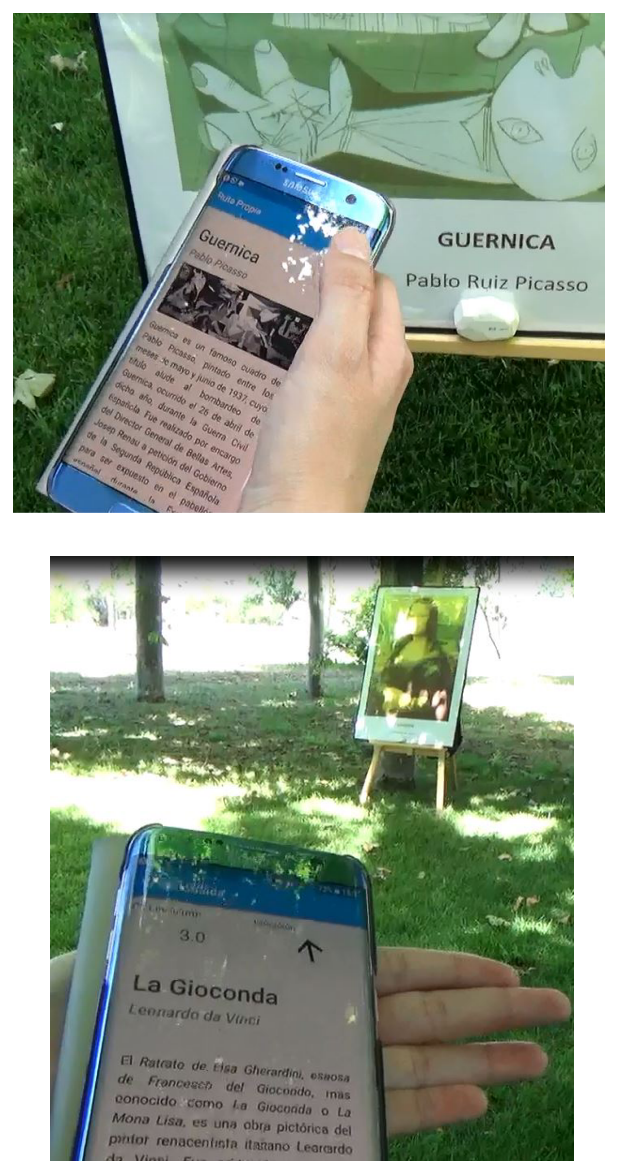

Figura 8: Arriba: Modo visita libre con información relativa a cuadro más próximo. Abajo: Visita guiada de cuadro a cuadro con indicador de a qué distancia y orientación encontrar un cuadro.

\section{Agradecimientos}

Los autores agradecen la financiación recibida de TARSIUS (TIN2015-71564-C4-2-R (MINECO/FEDER)), REPNIN (TEC2015-71426REDT) y Tomoloc (CSIC-PIE Ref. 201450E011).

\section{English summary}

\section{SMARTPHONE AUGMENTED INFORMATION FOR MUSEUMS WITH BLE LOCALIZATION}

\begin{abstract}
We present the design and development of a solution for a contextualized guidance during visits to museums. Several Bluetooth Low Energy (BLE) beacons have been distributed around the environment, from which the smartphone reads the Received Signal Strenght (RSS), together with information from the accelerometer and mag-
\end{abstract}


netometer sensors. We use the RSS data and the context information previously stored in the cloud, to estimate the smartphone's localization or pose of the person in the museum. The location is used to calculate the distance and orientation of the person with respect to the pictures of interest. The accuracy in the ability to measure these parameters is analyzed using different trilateration methods using extended Kalman filters (EKF) with loose and tightcoupled integration. The localization performance obtained allow us to have enough precision to be able to guide the user in search of a recommended picture, or also to give the information of the nearest frame in the user's viewing direction. The potential use and impact of this approach can be extended to other applications in industrial or robotic sectors that require instruments to search objects of interest.

Keywords: Localization, Signal strength, Bayes estimation, BLE beacons.

\section{Referencias}

[1] T. Aguilera, J. A. Paredes, F. J. Álvarez, J. I. Suárez, and A. Hernández. Acoustic local positioning system using an iOS device. 2013 International Conference on Indoor Positioning and Indoor Navigation, IPIN 2013, (October), 2013.

[2] Pascal Bihler, Paul Imhoff, and Armin B. Cremers. SmartGuide - A smartphone museum guide with ultrasound control. In Procedia Computer Science, volume 5, pages 586$592,2011$.

[3] Angelo Chianese, Fiammetta Marulli, Vincenzo Moscato, and Francesco Piccialli. SmARTweet: A location-based smart application for exhibits and museums. Proceedings - 2013 International Conference on SignalImage Technology and Internet-Based Systems, SITIS 2013, pages 408-415, 2013.

[4] Ramsey Faragher and Robert Harle. Location fingerprinting with bluetooth low energy beacons. IEEE Journal on Selected Areas in Communications, 33(11), 2015.
[5] Damian E. Grzechca, Piotr Pelczar, and Lukas Chruszczyk. Analysis of Object Location Accuracy for iBeacon Technology based on the RSSI Path Loss Model and Fingerprint Map. International Journal of Electronics and Telecommunications, 62(4):371-378, 2016.

[6] A. R. Jiménez and F. Seco. Finding objects using UWB or BLE localization technology: A museum-like use case. 2017 International Conference on Indoor Positioning and Indoor Navigation, IPIN 2017, 2017Janua(September):1-8, 2017.

[7] A. R. Jimenez, Fernando Seco Granja, José Carlos Prieto Honorato, Jorge I. Guevara Rosas, and A.R. Jiménez. Accurate Pedestrian Indoor Navigation by Tightly Coupling a Foot-mounted IMU and RFID Measurements. IEEE Transactions on Instrumentation and Measurement, 61(1):178-189, jan 2012.

[8] Markus Kouhne and Jurgen Sieck. LocationBased Services with iBeacon Technology. Proceedings - 2nd International Conference on Artificial Intelligence, Modelling, and Simulation, AIMS 2014, pages 315-321, 2014.

[9] Rainer Mautz. Indoor Positioning Technologies. PhD thesis, ETH Zurich, 2012.

[10] Sagar Patil, Ms Shraddha Limbekar, Ms Amruta Mane, and Ms Netra Potnis. Smart Guide - an approach to the Smart Museum using Android. International Research Journal of Engineering and Technology, 5(2), 2018.

[11] Irene Rubino, Jetmir Xhembulla, Andrea Martina, Andrea Bottino, and Giovanni Malnati. Musa: Using indoor positioning and navigation to enhance cultural experiences in a museum. Sensors (Switzerland), 13(12):17445-17471, 2013.

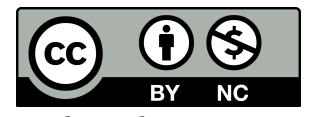

(C) 2018 by the authors. Submitted for possible open access publication under the terms and conditions of the Creative Commons Attribution CC-BY-NC 3.0 license (http://creativecommons.org/licenses/by-nc/3.0/). 